\title{
Stellar populations of local infrared-selected galaxies
}

\author{
X. Y. Chen ${ }^{1,2}$, Y. C. Liang ${ }^{1}$, F. Hammer ${ }^{3}$, \\ Y. H. Zhao ${ }^{1}$, \& G. H. Zhong ${ }^{1,2}$ \\ ${ }^{1}$ National Astronomical Observatories, Chinese Academy of Sciences, A20 Datun Road, \\ Chaoyang District, Beijing 100012, China; email: chenxy@nao.cas.cn; ycliang@nao.cas.cn \\ ${ }^{2}$ Graduate School of the Chinese Academy of Sciences, Beijing 100049, China \\ ${ }^{3}$ GEPI, Observatoire de Paris-Meudon, Meudon 92195, France
}

\begin{abstract}
The stellar populations of 849 local infrared-selected galaxies from SDSS and IRAS (including 419 star-forming galaxies, 326 composite galaxies, 35 Seyfert 2s, and 69 LINERs in 4 spectral classes) are studied by using STARLIGHT. Among the 4 spectral classes, the importance of young populations decreases from star-forming, composite, Seyfert 2 to LINER; and Seyfert 2 and LINER are more metal-rich; ULIGs (ultra luminous infrared galaxies) \& LIGs present the youngest populations among 3 infrared luminosity bins; and normal galaxies are more metal-rich. The dominant contributors to masses are all old populations.
\end{abstract}

Keywords. galaxies: evolution, galaxies: stellar content, infrared: galaxies

\section{Introduction}

Understanding the overall stellar population in galaxies is a crucial tool for unveiling the star formation and evolution of galaxies. Infrared-detected galaxies are one of the most interesting objects in the Universe and are related to the major star-forming process. We selected a large local sample of infrared-detected galaxies from SDSS optical spectra and IRAS infrared observations to study their stellar populations.

\section{Sample and spectral synthesis results}

We utilize a sample of 849 local infrared-selected galaxies from cross identifying between the main galaxy sample of SDSS DR4 and IRAS PSCz, and use the software STARLIGHT (Asari et al. 2007; Cid Fernandes et al. 2005) to fit the spectral absorptions and continua to study their stellar populations. The templates are the simple stellar populations (SSPs) from Bruzual \& Charlot 2003 (BC03) and spectra of star clusters. We further divided our sample into several sub-samples by two methods: 1) four spectral classes by using their emission-line ratios (Baldwin et al. 1981, BPT): 419 star-forming galaxies, 326 composite galaxies, 35 Seyfert 2s, and 69 LINERs; 2) three infrared luminosity bins (Elbaz et al. 2002): 299 ULIGs \& LIGs $\left(L_{I R} / L_{\odot}>10^{11}\right), 451$ starbursts $\left(10^{11}>L_{I R} / L_{\odot}>10^{10}\right)$, and 99 normal galaxies $\left(L_{I R} / L_{\odot}<10^{10}\right)$.

Throughout our synthesis, we use the Padova 1994 tracks (Alongi et al. 1993), the Chabrier (2003) IMF, the CAL reddening law (Calzetti et al. 1994), and 45 SSPs from BC03 (15 ages: $1 \mathrm{Myr} \sim 13 \mathrm{Gyr}, 3$ metallicities: $0.2,1.0,2.5 Z_{\odot}$ ). We further arrange the 15 ages of SSPs into 3 age bins: young with age $\leqslant 5 \times 10^{8} \mathrm{yr}$, old with age $\geqslant 1 \times 10^{10} \mathrm{yr}$, and intermediate-age populations with ages between these two. Fig. 1 shows the spectral fitting results for 4 spectral classes. We find that the importance of young populations decreases from star-forming, composite, Seyfert 2 to LINER, and LINER and Seyfert 2 are more metal-rich. Similar conclusions have been reached by Schawinski et al. (2007), 

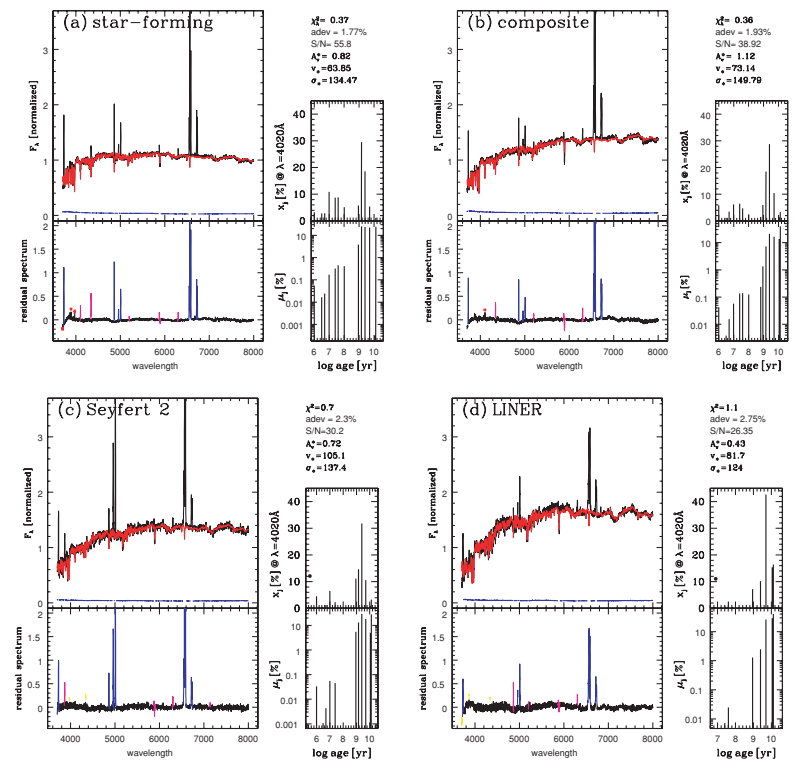

Figure 1. The spectral fitting results of 4 spectral classes: (a) star-forming galaxies (top-left 4 panels); (b) composite galaxies; (c) Seyfert 2s and (d) LINERs. In each class, top-left panel: observed spectra (black line), synthesis spectra (red line), and error spectra (green line); bottom-left panel: residual spectra (black line) and mask regions (color lines and points); the fraction of light (top-right) and of mass (bottom-right) associated to each age of 15 SSPs.

Stasińska et al. (2008) and Cid Fernandes et al. (2009). As for the different infrared luminosity bins, ULIGs \& LIGs present the youngest populations, and normal galaxies are more metal-rich. However, the dominant contributors to mass are old stellar populations in all sub-samples. Additionally, we also use 15 spectra of star clusters with different ages and metallicities given in Bica \& Alloin (1986a,b) to re-fit the combined spectra of each sub-sample, and draw consistent conclusions (see details in Chen et al. 2009).

\section{Acknowledgements}

We thank the NSFC grant support under Nos. 10933001, 109730

06, 10973015, 10673002, and the National Basic Research Program of China (973 Program) Nos.2007CB815404, 2007CB815406.

\section{References}

Alongi, M., Bertelli, G., Bressan, A. et al. 1993, A\& $A S, 97,851$

Asari, N. V., Cid Fernandes, R., Stasińska, G. et al. 2007, MNRAS, 381, 263

Baldwin, J. A., Phillips, M. M., \& Terlevich, R. 1981, PASP, 93, 5

Bica, E. \& Alloin, D. 1986a, A\&A A, 162, 21

Bica, E. \& Alloin, D. 1986b, $A \mathscr{S} A S, 66,171$

Bruzual, A. G., \& Charlot, S. 2003, MNRAS, 344, 1000

Calzetti, D., Kinney, A. L., \& Storchi-Bergmann, T. 1994, ApJ, 429, 582

Chabrier, G. 2003, PASP, 115, 763

Chen, X. Y., Liang, Y. C., Hammer, F., Zhao, Y. H., Zhong, G. H. 2009, A\& A, 495, 457

Cid Fernandes, R., Mateus, A., Sodré, L. et al. 2005, MNRAS, 358, 363

Cid Fernandes, R. et al. 2009, Rev. Mexicana AyA, 35, 127

Elbaz, D., Cesarsky, C. J., Chanial, P. et al. 2002, A\&A, 384, 848

Schawinski, K., Thomas, D., Sarzi, M. et al. 2007, MNRAS, 382, 1415

Stasinska, G., Asari, N. V., Cid Fernandes, R. et al. 2008, MNRAS, 391, 29 